\title{
MIPSS70+ v2.0 predicts long-term survival in myelofibrosis after allogeneic HCT with the Flu/Mel conditioning regimen
}

\author{
Haris Ali, ${ }^{1, *}$ Ibrahim Aldoss, ${ }^{1, \star}$ Dongyun Yang, ${ }^{2}$ Sally Mokhtari, ${ }^{3}$ Samer Khaled, ${ }^{1}$ Ahmed Aribi, ${ }^{1}$ Michelle Afkhami, ${ }^{4}$ Monzr M. Al Malki, ${ }^{1}$ \\ Thai Cao, ${ }^{1}$ Matthew Mei, ${ }^{1}$ Margaret O'Donnell, ${ }^{1}$ Amandeep Salhotra, ${ }^{1}$ Vinod Pullarkat, ${ }^{1}$ Lixin Yang, ${ }^{4}$ Anthony S. Stein, ${ }^{1}$ Guido Marcucci, ${ }^{1}$ \\ Stephen J. Forman, ${ }^{1}$ Ryotaro Nakamura, ${ }^{1}$ Raju Pillai, ${ }^{4,+}$ and David Snyder ${ }^{1,+}$ \\ ${ }^{1}$ Department of Hematology and Hematopoietic Cell Transplantation, ${ }^{2}$ Division of Biostatistics, Department of Information Sciences, ${ }^{3}$ Department of Clinical Translational \\ Program Development, and ${ }^{4}$ Department of Pathology, City of Hope, Duarte, CA
}

\section{Key Points}

- Favorable long-term OS and low relapse rates were achieved with allo-HCT and a Flu/Mel regimen in patients with MF.

- Both MIPSS70 and MIPSS70+ v2.0 scores can predict outcomes after allo-HCT.
Although allogeneic hematopoietic cell transplantation (allo-HCT) is the only curative treatment for myelofibrosis (MF), data are limited on how molecular markers predict transplantation outcomes. We retrospectively evaluated transplantation outcomes of 110 consecutive MF patients who underwent allo-HCT with a fludarabine/melphalan (Flu/Mel) conditioning regimen at our center and assessed the impact of molecular markers on outcomes based on a 72-gene next-generation sequencing panel and Mutation-Enhanced International Prognostic Scoring System 70+ v2.0 (MIPSS70+ v2.0). With a median follow-up of 63.7 months, the 5-year overall survival (OS) rate was $65 \%$ and the nonrelapse mortality (NRM) rate was 17\%. In mutational analysis, JAK2 V617F and ASXL1 mutations were the most common. By univariable analysis, higher Dynamic International Prognostic Scoring System scores, unrelated donor type, and very-high-risk cytogenetics were significantly associated with lower OS. Only CBL mutations were significantly associated with lower OS (hazard ratio [HR], 2.64; $P=.032$ ) and increased NRM (HR, 3.68; $P=.004$ ) after allo-HCT, but CALR, ASXL1, and IDH mutations did not have an impact on transplantation outcomes. Patient classification per MIPSS70 showed worse OS for high-risk (HR, 0.49; $P=.039$ ) compared with intermediate-risk patients. Classification per MIPSS70+ v2.0 demonstrated better OS when intermediate-risk patients were compared with high-risk patients (HR, 0.291) and much lower OS when very-high-risk patients were compared with high-risk patients (HR, 5.05; $P \leq .001$ ). In summary, we present one of the largest single-center experiences of Flu/Mel-based allo-HCT, demonstrating that revised cytogenetic changes and MIPSS70+ v2.0 score predict transplantation outcomes, and thus can better inform physicians and patients in making decisions about allo-HCT.

\section{Introduction}

Primary myelofibrosis (MF) is a classic Philadelphia chromosome-negative myeloproliferative neoplasm characterized by clonal proliferation of hematopoietic stem cells with marrow fibrosis, splenomegaly, cytopenias, and profound constitutional symptoms from aberrant and excessive cytokine production. Secondary MF can develop in patients with prior polycythemia rubra vera and essential thrombocythemia and can be clinically and pathologically indistinguishable from primary MF. ${ }^{1}$ Because of its variable clinical course, several prognostic models have been developed in the past few decades to predict patients' survival outcomes, with earlier models (ie, International Prognostic Scoring System,
Submitted 27 September 2018; accepted 7 December 2018. DOI 10.1182/ bloodadvances.2018026658.

*H.A. and I.A. contributed equally to this article as first authors.

tR.P. and D.S. contributed equally to this article as senior authors.
The full-text version of this article contains a data supplement. C 2019 by The American Society of Hematology 
Lille Scoring System, and Dynamic International Prognostic Scoring System [DIPSS]) using only clinical characteristics for risk stratification. ${ }^{2,3}$ Because certain cytogenetic abnormalities can predict worse survival, DIPSS Plus was developed by adding cytogenetics to the DIPSS prognostic model. ${ }^{4}$ After the discovery of JAK2 mutations in 2005, several frequent driver and nondriver mutations were detected in MF patients ${ }^{5}$; driver mutations (JAK2, $C A L R$, and $M P L)$ were found in $90 \%$ of them. ${ }^{6}$ Triple-negative patients who did not carry driver mutations had worse outcomes. ${ }^{6}$ Several other recurrent mutations ( $A S X L 1, E Z H 2, I D H, S R S F 2$, $S F 3 B 1, U 2 A F I$, and $C B L$ ) have been detected in triple-negative patients and in patients with 1 of the 3 driver mutations. To predict outcomes of patients age 70 years or younger, Mutation-Enhanced International Prognostic Scoring System 70 (MIPSS70) was developed by incorporating all the key clinical characteristics, cytogenetics, and mutational factors into a single system. ${ }^{1}$ MIPSS70 was recently revised as MIPSS70+ v2.0 with refinements in degrees of anemia, cytogenetics, and high molecular risk (HMR) category, based on the presence of the 5 prognostically detrimental mutated genes (ASXL1, $E Z H 2, S R S F 2, I D H 1 / 2$, and U2AF1). ${ }^{7}$ Cytogenetics risk stratification has recently been revised by Tefferi et al. ${ }^{8}$ This new and improved cytogenetics risk stratification model expanded the previous 2-sided (favorable and unfavorable) risk categories into a 3-tiered model of favorable, unfavorable, and very-high-risk (VHR) categories. ${ }^{8}$

Although several therapeutic options are available for disease management (ie, ruxolitinib), allogeneic hematopoietic cell transplantation (allo-HCT), despite its significant risk of mortality and morbidities, is still the only curative option for MF. We and others have reported promising results for outcomes in MF patients after allo-HCT, ${ }^{9-13}$ and with the development of reduced-intensity conditioning (RIC), the number of allo-HCTs for MF has been steadily increasing over time. ${ }^{14}$ However, no standard conditioning regimen or prophylaxis for graft-versus-host disease (GVHD) has yet been established for MF, and limited data are available regarding the impact of somatic mutations on transplantation outcomes in MF. A recent study by Kröger et $\mathrm{al}^{5}$ retrospectively evaluated 169 patients who received a transplant with busulfan-based conditioning. They were screened for 16 mutations common in MF, and results showed that a CALR mutation was associated with improved overall survival (OS), whereas older age, transformation to acute myeloid leukemia (AML), and DIPSS intermediate-2 risk and high risk were associated with poor OS in multivariable analysis. ${ }^{5}$ Herein, we evaluated the outcome of allo-HCT in MF patients who uniformly received fludarabine/melphalan (Flu/Mel) conditioning at City of Hope $(\mathrm{COH})$ and assessed the impact of somatic mutations on transplantation outcomes based on a 72-gene next-generation sequencing (NGS) panel and MIPSS70+ v2.0.

\section{Methods}

\section{Study population}

From a total of 145 patients with primary or secondary MF (excluding those who transformed to $\mathrm{AML}$ ) who received allo-HCT at $\mathrm{COH}$ from January 2004 to February 2017, 110 patients underwent RIC transplantation with fludarabine $\left(25 \mathrm{mg} / \mathrm{m}^{2}\right.$ for 5 days for a total of $125 \mathrm{mg} / \mathrm{m}^{2}$ ) in combination with melphalan (at a total dose of $100 \mathrm{mg} / \mathrm{m}^{2}$ or $140 \mathrm{mg} / \mathrm{m}^{2}$ ) as the conditioning regimen. This retrospective study was approved by the $\mathrm{COH}$ Institutional Review Board per the Declaration of Helsinki.

\section{Data collection}

Patients' prior medical history, demographic information, cytogenetic and molecular data, prior treatments, and transplantation outcomes were collected through the institution's electronic medical records, medical record reviews, and the Blood and Marrow Transplant Program database. DIPSS scores for each patient was generated at the time of HCT based on age, hemoglobin, white blood cell count, percentage of myeloblasts in the peripheral blood, and constitutional symptoms. MIPSS70 and MIPSS70+ v2.0 scores were generated for 93 patients who had an available DNA sample that was tested before allo-HCT.

\section{NGS library preparation and bioinformatics analysis}

NGS libraries were prepared from genomic DNA (40 ng) using the SureSelect Target Enrichment System (Agilent Technologies, Inc.) after transposase-based fragmentation and adapter ligation. The adapter-ligated library was amplified by polymerase chain reaction, and quality control was performed for sizing and concentration. Target regions were captured by using a customized SureSelect library (Agilent Technologies, Inc.) for all coding exons plus 10 flanking bases of 72 genes as previously described ${ }^{15}$ (supplemental Table 1). After hybridization of $750 \mathrm{ng}$ of adapter-ligated library with biotinlabeled probes specific to target regions, the dual-index tag was added during postcapture amplification by polymerase chain reaction. The amplified captured libraries used a high-sensitivity DNA Bioanalyzer kit (Agilent Technologies, Inc.) for quality control, and they were then pooled and sequenced using Miseq V2 Reagent Kit for 300 cycles with 150-bp paired-end sequencing. Alignment of sequence reads to the human genome (GRCh37/hg19), variant calling, and annotation were performed independently using 2 software applications (CLC Biomedical Workbench; CLC Bio, Aarhus, Denmark and NextGENe; SoftGenetics, State College, PA). Annotated variants were processed by using previously published criteria. ${ }^{16,17}$ Synonymous variants, variants located $>2$ bp outside protein-coding regions, polymorphisms present in $>1 \%$ in population databases, including ExaC, Exome Variant Server, and the 1000 Genomes Project, and variants with $<30 \times$ coverage were filtered. The remaining variants were evaluated by using tumor-specific databases (COSMIC, cBioportal), information retrieved from literature, sequence conservation, and in silico prediction algorithms, including SIFT, Polyphen-2, and FATHMM, for clinical significance.

\section{Definitions of outcomes}

OS was defined as the time from the day of transplantation to death as a result of any cause. Patients who were alive at their last followup were censored. Death from causes other than relapse was considered nonrelapse mortality (NRM). Relapse was defined as the time to onset of recurrent MF or AML, which was determined by morphologic evidence in bone marrow or extramedullary sites. Relapse-free survival (RFS) was defined as the time to relapse or death from any cause, whichever came first. Acute and chronic GVHD were graded according to previously published criteria. ${ }^{18,19}$ The composite end point of GVHD-free RFS included grade III to IV acute GVHD, chronic GVHD that required systemic therapy, relapse, or death in the first year after $\mathrm{HCT}^{20}$

\section{Statistical analysis}

Patients' characteristics and disease- and transplant-related variables were summarized with descriptive statistics. OS and RFS were 
computed using the Kaplan-Meier method, and differences were compared using a log-rank test. When calculating NRM, relapse was counted as a competing risk. For patients who relapsed but were alive, the last contact was used as the latest follow-up. Similarly, the cumulative incidence of relapse was calculated with death before relapse as a competing risk event. Cumulative incidence of relapse and NRM were estimated and the differences were compared using the Gray method. Cox proportional hazards models were used for univariable and multivariable analyses of OS and RFS, and the hazard ratios (HRs) are reported with 95\% confidence intervals (Cls). Baseline patient demographic, disease, and transplant variables that were significantly associated with OS in the univariable analyses at one level were selected as covariates in the multivariable analyses. The proportional subdistribution hazards model for competing risks was used to calculate HRs and $95 \% \mathrm{Cls}$ for relapse and NRM.

All reported $P$ values were 2 -sided and were not adjusted for testing multiple hypotheses. A significance level of 0.05 was used. Statistical analyses were performed using SAS 9.4/STAT 14.1 (SAS Institute, Cary, NC).

\section{Results}

\section{Patient characteristics}

Patient baseline and transplant characteristics are summarized in Table 1. Briefly, median age at diagnosis and allo-HCT were 55 years (range, 29-72 years) and 59 years (range, 38-72 years), respectively. Intermediate- 2 and high risk by DIPSS accounted for 83 patients $(76 \%)$ at the time of transplantation. Splenectomy was performed in 16 patients (15\%), and ruxolitinib was used in 32 patients (29\%) before transplantation. Patients received allo-HCT from a matched related donor (MRD; $n=51$ [46\%]), a 10/10 matched unrelated donor (MUD: $\mathrm{n}=44$ [40\%]), or a $<10 / 10$ allele or antigen-matched unrelated donor (mMUD; $n=15$ [14\%]). Tacrolimus/sirolimus (Tac/Sir)-based GVHD prophylaxis was used in 100 patients (91\%).

\section{Cytogenetics}

We evaluated the impact of cytogenetic changes $(n=106)$ that were based on recently developed revised cytogenetic risk stratification on transplant outcomes by Tefferi et al ${ }^{8}$ and identified 67 patients $(61 \%)$ in favorable, 24 patients $(22 \%)$ in unfavorable, and 15 patients (14\%) in very-high-risk (VHR) groups. (Table 1)

\section{Molecular profile}

Among the 93 patients who had available DNA samples before allo$\mathrm{HCT}$, a median of 2 mutations were detected with at least 1 mutation in $95 \%$ of patients $(n=88)$. JAK2 V617F was the most common alteration noted in 54 patients (58.1\%) (Figure 1A). Other common mutations were $A S X L 1$ ( $\mathrm{n}=41$ [44\%]), CALR type 1 ( $\mathrm{n}=15$ [16.1\%]), TET2 ( $\mathrm{n}=12[13 \%]), S R S F 2$ and DNMT3A (each $\mathrm{n}=10[11 \%]) . U 2 A F 1$ and $C B L$ (each $\mathrm{n}=8[8.6 \%]), I D H 1 / 2$ ( $n=5[5.4 \%]), \operatorname{TP5} 3(n=4[4.3 \%])$, and CALR type $2(n=3[3.2 \%])$. No detectable mutations were found in 5 patients (5.4\%) (Figure $1 \mathrm{~A}$ )

Most of the genomic alterations were detected in pathways involved in signal transduction, histone modification, DNA methylation, and RNA splicing factors (Figure 1B), and $A S X L 1$ mutations were seen at a higher frequency (44\%) than previously reported (Figure 1B). Most ASXL1 alterations were loss-of-function mutations in exons
12 and 13 , and a frameshift alteration at codon 646 had the highest frequency (Figure 1C).

HMR genes ( $A S L X 1, E Z H 2, I D H 1 / 2, S R S F 2$, and U2AF1) were identified in 48 patients (52\%), with 30 patients (32\%) carrying 1 and 18 patients (19\%) carrying more than 1 HMR mutation (Figure 1B).

\section{Allo-HCT outcomes}

All but 2 patients engrafted, with the median time of 16 days (range, 10-33 days) for neutrophil engraftment and 35 days (range, 11-226 days) for platelet engraftment (supplemental Figure 1A-B). Median follow-up time for surviving patients was 63.7 months (range, 11.9158.5 months). The 5-year OS for the entire cohort was $65 \%$ (95\% $\mathrm{Cl}, 54 \%-73 \%)$ and progression-free survival was $60 \%(95 \% \mathrm{Cl}$, 50\%-69\%) (supplemental Figure 1C). Cumulative incidence of relapse and NRM at 5 years were $17 \%(95 \% \mathrm{Cl}, 10 \%-24 \%)$ and $24 \%(95 \% \mathrm{Cl}, 16 \%-32 \%)$, respectively (supplemental Figure 1D). Cumulative incidence of acute GVHD grades II to IV and III to IV by day 100 were $45 \%$ and $17 \%$, respectively, and cumulative incidence of chronic extensive GVHD at 12 and 36 months was $45 \%$ and $59 \%$, respectively. GVHD-free RFS was $41 \%$ at 12 months and $34 \%$ at 60 months (supplemental Figure 1C).

\section{Clinical prognostic factors for survival}

In univariable analysis, higher DIPSS scores at the time of transplantation, donor type (<10/10 mMUD) (Figure 2A), and VHR cytogenetics (Figure 2B) were significantly associated with poor OS with a trend for male patients showing lower OS $(P=.07)$ (Table 2). In multivariable analysis, although DIPSS scores lost significance, female sex (HR, 0.37; 95\% Cl, 0.18-0.74; $P=.005)$, mMUD (HR, 8.32; 95\% Cl, 3.47-19.93; $P<.001$ ), and VHR cytogenetics (HR, 4.19;95\% Cl, 1.93-9.13; $P=.001)$ remained significant. Among these significant variables for OS, mMUD was associated with increased NRM (HR, 5.64; 95\% Cl, 2.20-14.45; $P<.001$ ) but not relapse by univariable analysis (Table 3 ). In contrast, VHR cytogenetics was predictive of relapse (HR, 2.95; $95 \% \mathrm{Cl}, 1.11-7.82 ; P=.035)$ and not NRM.

\section{Somatic mutation impact on HCT outcomes}

We next evaluated the effect of somatic mutations on survival outcome after allo-HCT and found that triple-negative status $(P=.063), \operatorname{HMR}(P=.73)$, and $>1 \operatorname{HMR}(P=.59)$ did not have a significant impact on survival after HCT when triple-negative, non-HMR, and $1 \mathrm{HMR}$ were used as reference points, respectively (supplemental Figure 2). Contrary to a previous report, ${ }^{5} C A L R$ type $1(P=.42)$ and $A S X L 1(P=.29)$ mutations also did not have an impact on survival after HCT in our cohort. Only $C B L$ mutations were significantly associated with lower OS (HR, $2.64 ; 95 \% \mathrm{Cl}, 1.09-6.38 ; P=.032)$ and lower disease-free survival (DFS) (HR, 4.35; 95\% Cl, 1.83-10.36; $P<.001$ ), largely attributable to increased NRM (HR, 3.68; $95 \% \mathrm{Cl}, 1.45-$ 9.35; $P=.004)$. In addition, U2AF1 mutations were significantly associated with NRM (HR, 3.42; 95\% Cl, 1.50-7.80; $P=.009)$ (Figure 2C-D).

\section{MIPSS70 and MIPSS70+ v2.0}

We next measured MIPSS70 and MIPSS70+ v2.0 scores in 93 patients with available cytogenetics and molecular mutations, and we found 72 patients (77\%) with high risk and 21 patients 


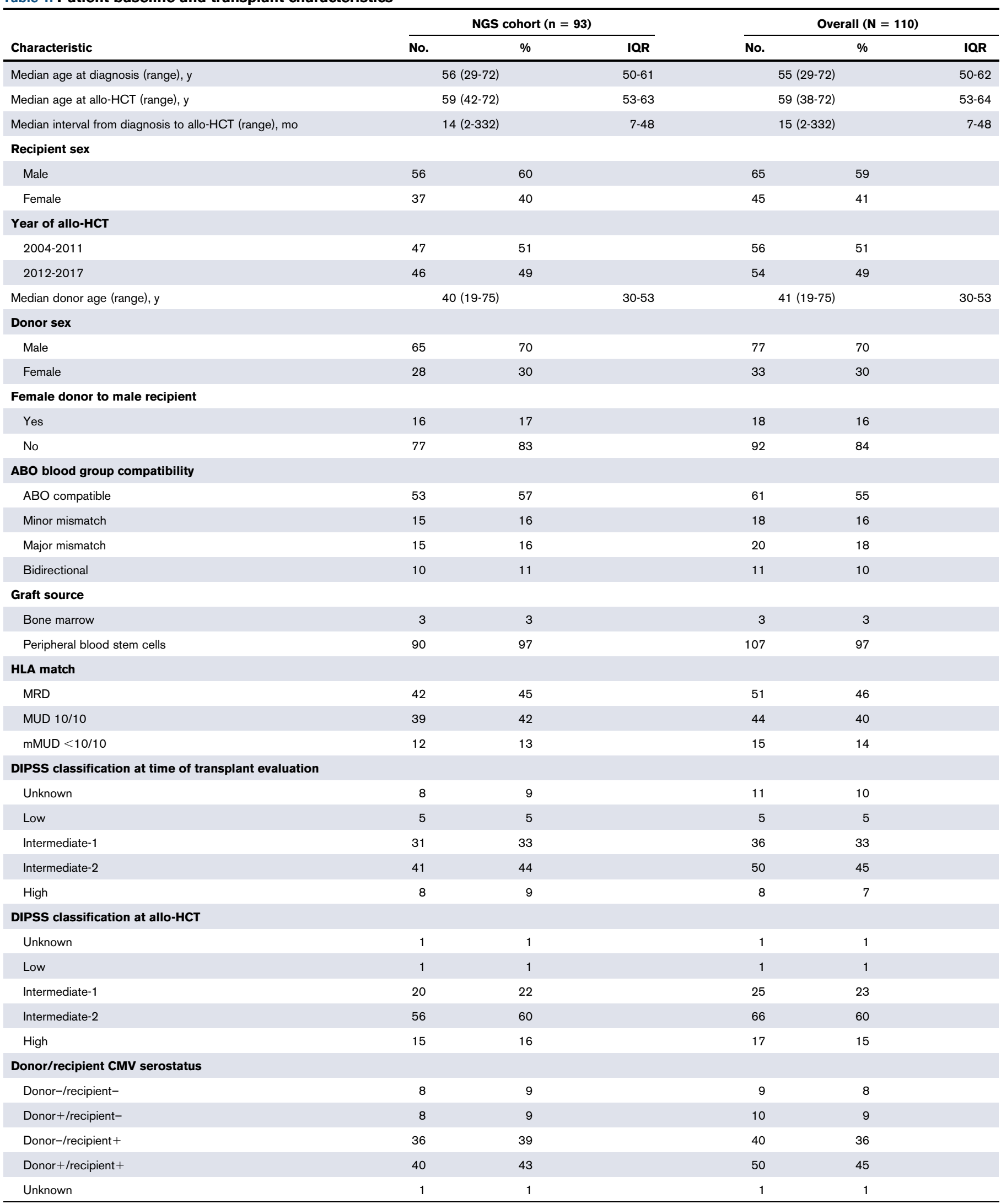

CMV, cytomegalovirus; IQR, interquartile range. 


\begin{tabular}{|c|c|c|c|c|c|c|}
\hline \multirow[b]{2}{*}{ Characteristic } & \multicolumn{3}{|c|}{ NGS cohort $(n=93)$} & \multicolumn{3}{|c|}{ Overall $(\mathrm{N}=110)$} \\
\hline & No. & $\%$ & IQR & No. & $\%$ & IQR \\
\hline \multicolumn{7}{|l|}{ Karnofsky performance status, $\%$} \\
\hline $90-100$ & 37 & 40 & & 46 & 42 & \\
\hline $70-80$ & 32 & 34 & & 37 & 34 & \\
\hline Unknown & 24 & 26 & & 27 & 25 & \\
\hline Median HCT comorbidity index (range) & & & $0-3$ & & & $0-2$ \\
\hline 0 & 32 & 34 & & 38 & 35 & \\
\hline $1-2$ & 21 & 23 & & 26 & 24 & \\
\hline$>2$ & 18 & 19 & & 21 & 19 & \\
\hline Unknown & 22 & 24 & & 25 & 23 & \\
\hline \multicolumn{7}{|l|}{ GVHD prophylaxis } \\
\hline Tacrolimus/sirolimus based & 84 & 90 & & 100 & 91 & \\
\hline Tacrolimus based & 4 & 4 & & 4 & 4 & \\
\hline Cyclosporine/CellCept based & 5 & 5 & & 6 & 5 & \\
\hline \multicolumn{7}{|l|}{ Type of MF } \\
\hline Primary MF & 51 & 55 & & 58 & 53 & \\
\hline Secondary polycythemia vera & 15 & 16 & & 19 & 17 & \\
\hline Secondary essential thrombocythemia & 27 & 29 & & 33 & 30 & \\
\hline Secondary MF & 42 & 45 & & 52 & 47 & \\
\hline \multicolumn{7}{|l|}{ Ruxolitinib use } \\
\hline No & 66 & 71 & & 78 & 71 & \\
\hline Yes & 27 & 29 & & 32 & 29 & \\
\hline \multicolumn{7}{|l|}{ Splenectomy before allo-HCT } \\
\hline No & 81 & 87 & & 94 & 85 & \\
\hline Yes & 12 & 13 & & 16 & 15 & \\
\hline \multicolumn{7}{|l|}{ Cytogenetics } \\
\hline Unknown & 3 & 3 & & 4 & 4 & \\
\hline Favorable & 57 & 61 & & 67 & 61 & \\
\hline Unfavorable & 20 & 22 & & 24 & 22 & \\
\hline VHR & 13 & 14 & & 15 & 14 & \\
\hline \multicolumn{7}{|l|}{ MIPSS70+ v2.0 classification } \\
\hline Unknown & 0 & & & 17 & 15 & \\
\hline Intermediate risk & 11 & 12 & & 11 & 10 & \\
\hline High risk & 47 & 51 & & 47 & 43 & \\
\hline VHR & 35 & 38 & & 35 & 32 & \\
\hline
\end{tabular}

CMV, cytomegalovirus; IQR, interquartile range.

(23\%) with intermediate risk per MIPSS70 classification. The MIPSS70 high-risk group had worse OS (HR, 3.49; 95\% $\mathrm{Cl}, 1.07-11.39 ; P=.039$ ), and DFS (HR, 4.58; 95\% Cl, 1.40$14.91 ; P=.012)$ compared with the intermediate-risk group (Figure 3A-B).

Per MIPSS70+ v2.0, patients were classified into intermediate risk $(n=11)$, high risk $(n=47)$, or VHR $(n=35)$. MIPSS70+ v2.0 scores predicted OS, DFS, and NRM. Compared with the high-risk group, intermediate-risk patients had better OS (HR, 0.291; 95\% Cl, 0.04-2.26) and DFS (HR, 0.24; 95\% Cl, 0.03-1.91), whereas the VHR group had much lower OS $(\mathrm{HR}, 5.05 ; 95 \% \mathrm{Cl}$,
2.39-10.74; $P \leq .001)$ and DFS (HR, 3.87; 95\% Cl, 1.90-7.88; $P<.001$ ) (Figure 3C-D). Compared with the high-risk group, the intermediate-risk group had lower ( $\mathrm{HR}, 0.51 ; 95 \% \mathrm{Cl}, 0.06-4.23$ ) and the VHR group had higher $(\mathrm{HR}, 3.24 ; 95 \% \mathrm{Cl}, 1.47-7.13$; $P=.004)$ NRM.

We next compared patients' distribution by MIPSS70 vs DIPSS and MIPSS70+ v2.0 vs DIPSS scores (supplemental Figure 3) and found that only $24 \%$ of patients $(n=18)$ categorized as intermediate- 1 or intermediate- 2 by the DIPSS were present in the intermediate-risk group by MIPSS70, and the remaining $76 \%(n=58)$ were categorized as high risk by MIPSS70 


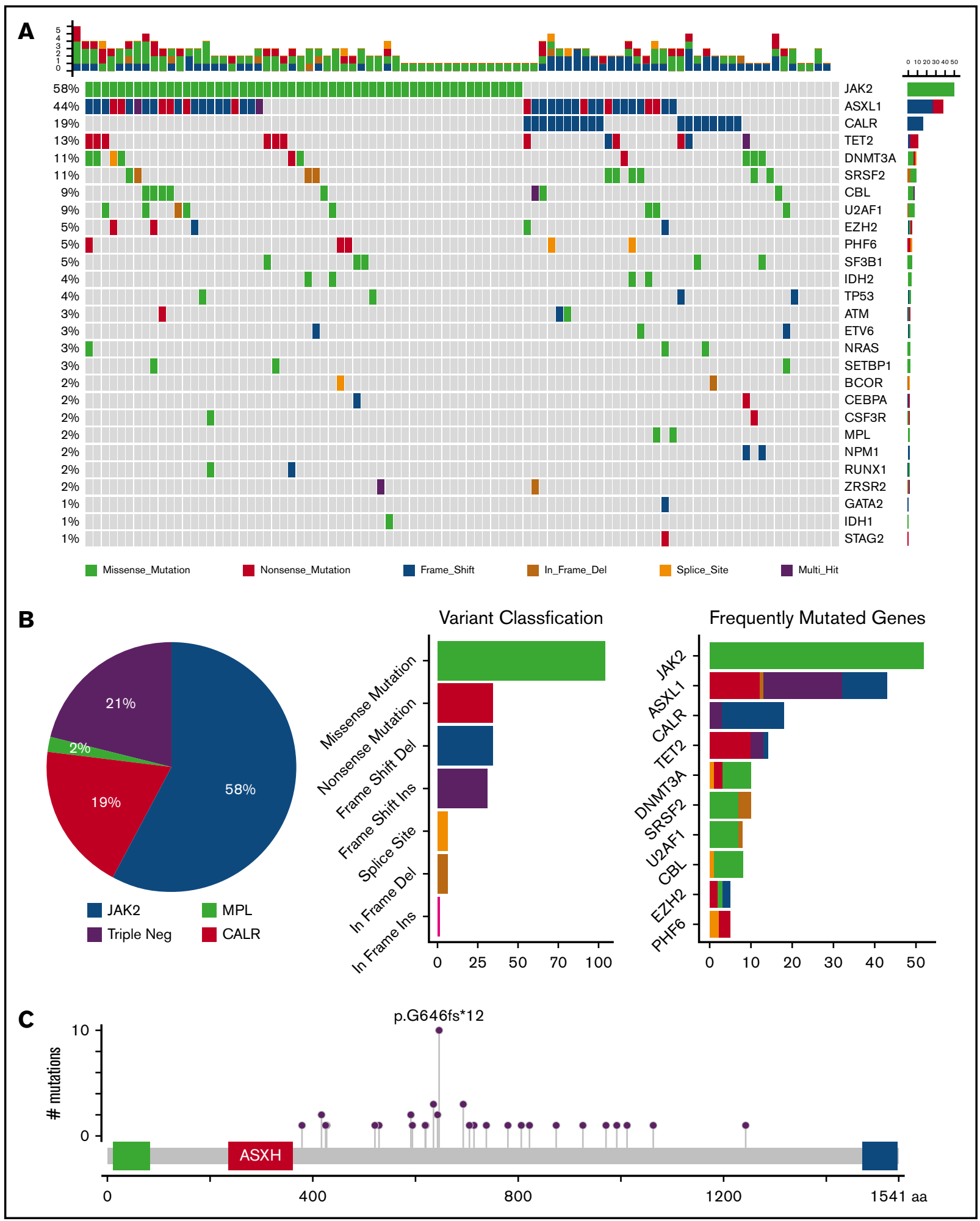

Figure 1. Molecular profile of MF. (A) Mutations visualized as an oncoplot. Each column represents a sample and each row represents a different gene. The top barplot has the frequency of mutations for each patient, and the right-hand barplot has the frequency of mutations in each gene. By default, samples are ordered by the most mutated genes. (B) Mutational frequency of driver mutations and triple-negative (Triple Neg) mutations (pie chart), variant classification (first barplot), and frequently mutated genes per variant classifications (second barplot). (C) ASXL1 alterations. 


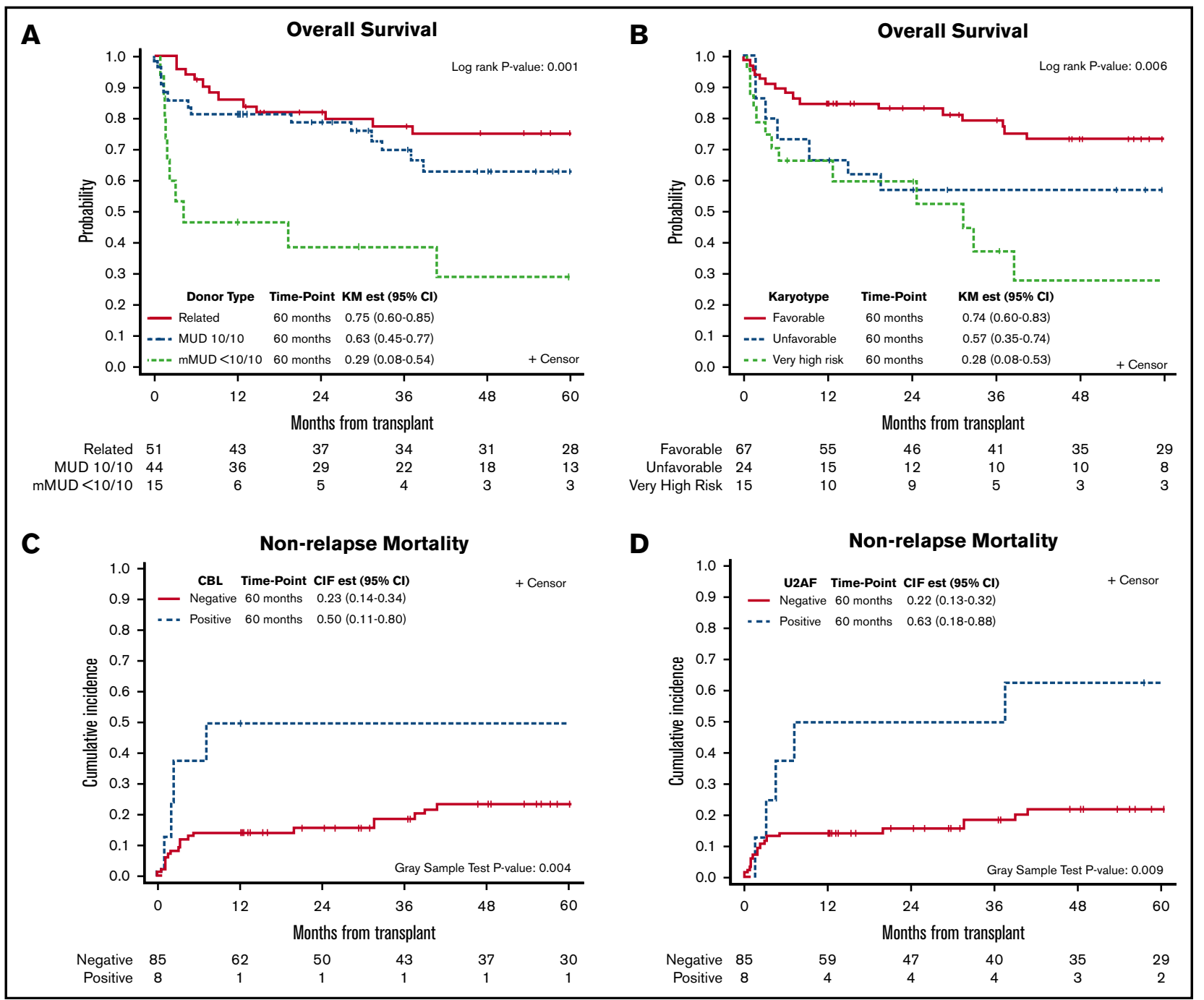

Figure 2. Kaplan-Meier curves defining survival outcomes at 60 months after allo-HCT. Overall survival based on donor type and cytogenetics (A-B); impact of $C B L$ and U2AF mutations on NRM (C-D). CIR est, cumulative incidence of relapse estimate; KM est, Kaplan-Meier estimate.

classification. However, of patients categorized as high risk by DIPSS ( $n=15)$, the majority $(n=13[87 \%])$ were also in the high-risk category by MIPSS70. Similarly, among the 76 patients categorized as intermediate- 1 or intermediate- 2 risk by the DIPSS, only 11 patients (14.5\%) were intermediate risk when MIPSS70+ v2.0 was used, and the rest were categorized as high risk ( $n=41$ [54.0\%]) or VHR $(n=24$ [31.5\%]) by the MIPSS70+ v2.0. High-risk patients by the DIPSS $(n=15)$ were categorized as high risk ( $n=4$ $[26.6 \%])$ or VHR $(n=11[73.4 \%])$ by the MIPSS70+ v2.0.

\section{Discussion}

Historically, therapy for MF has focused on symptomatic management with various agents (JAK2 inhibitors, androgens, erythrocytestimulating agents, cytoreductive agents) and supportive measures (red blood cell transfusions, steroids). However, regardless of the transplant-related morbidity and mortality, allo-HCT remains the only curative option for these patients. The number of allo-HCTs performed for MF patients is steadily increasing over time ${ }^{14}$ and patient's age at transplantation does not have an impact on transplant outcomes ${ }^{10}$ (median age at diagnosis is $\sim 65$ years) ${ }^{21}$

We previously presented favorable outcomes of transplantation in the MF population that used Flu/Mel RIC with Tac/Sir as GVHD prophylaxis. $^{22,23}$ Rondelli et $\mathrm{al}^{13}$ previously presented outcomes of a prospective phase 2 study, the Myeloproliferative Disorder Consortium 101 trial (MPD-RC 101) in 66 patients with primary MF undergoing allo-HCT with Flu/Mel (sibling donors) or Flu/Mel and antithymocyte globulin (unrelated donors). The study showed inferior OS and NRM in the MUD cohort. These results were in contrast with our study in which we did not see any differences between our MUD and MRD cohorts. However, mMUD was associated with poor survival. Lower survival outcomes might be explained by the use of antithymocyte globulin as part of the conditioning regimen for MUD in MPD-RC 101, resulting in lower engraftment rate and higher risk of secondary graft failure. Here we present long-term outcomes (median follow-up $>5$ years) of 110 patients treated with Flu/Mel conditioning at our center. This study, although not prospective, in agreement with other studies, ${ }^{9,10}$ shows 


\begin{tabular}{|c|c|c|c|c|c|c|c|c|c|c|c|c|c|}
\hline \multirow[b]{2}{*}{ Characteristic } & \multirow[b]{2}{*}{ No. } & \multicolumn{3}{|c|}{ os } & \multicolumn{3}{|c|}{ DFS } & \multicolumn{3}{|c|}{ Relapse } & \multicolumn{3}{|c|}{ NRM } \\
\hline & & $\mathbf{H R}^{*}$ & $95 \% \mathrm{Cl}$ & $\boldsymbol{P}^{*}$ & $\mathbf{H R}^{*}$ & $95 \% \mathrm{Cl}$ & $\boldsymbol{P}^{*}$ & HRt & $95 \% \mathrm{Cl}$ & $P+$ & HRt & $95 \% \mathrm{Cl}$ & $P+$ \\
\hline \multicolumn{14}{|l|}{ Age at diagnosis, $y$} \\
\hline$<55$ & 55 & Ref & & .71 & Ref & & .96 & Ref & & .32 & Ref & & .48 \\
\hline $55-65$ & 43 & 1.20 & $0.62-2.31$ & & 0.92 & $0.50-1.69$ & & 0.51 & $0.18-1.43$ & & 1.47 & $0.66-3.25$ & \\
\hline$>65$ & 12 & 1.46 & $0.54-3.92$ & & 0.97 & $0.37-2.54$ & & 0.38 & $0.05-3.04$ & & 1.76 & $0.62-5.00$ & \\
\hline \multicolumn{14}{|l|}{ Age at allo-HCT, y } \\
\hline$<55$ & 38 & Ref & & .24 & Ref & & .88 & Ref & & .30 & Ref & & .14 \\
\hline $55-65$ & 51 & 1.60 & $0.77-3.33$ & & 1.13 & $0.59-2.16$ & & 0.46 & $0.16-1.28$ & & 2.36 & $0.90-6.16$ & \\
\hline$>65$ & 21 & 2.03 & $0.83-4.95$ & & 1.22 & $0.53-2.81$ & & 0.56 & $0.16-2.00$ & & 2.37 & $0.75-7.44$ & \\
\hline \multicolumn{14}{|c|}{ Years from diagnosis to HCT } \\
\hline$<1$ & 47 & Ref & & .71 & Ref & & .60 & Ref & & .63 & Ref & & .63 \\
\hline 1 to $<3$ & 29 & 0.72 & $0.33-1.58$ & & 0.71 & $0.35-1.46$ & & 0.86 & $0.29-2.54$ & & 0.65 & $0.25-1.72$ & \\
\hline $3+$ & 34 & 0.91 & $0.45-1.87$ & & 0.80 & $0.40-1.57$ & & 0.57 & $0.18-1.81$ & & 1.00 & $0.44-2.26$ & \\
\hline \multicolumn{14}{|l|}{ Sex } \\
\hline Male & 65 & Ref & & .071 & Ref & & .065 & Ref & & .20 & Ref & & .26 \\
\hline Female & 45 & 0.54 & $0.28-1.07$ & & 0.56 & $0.30-1.05$ & & 0.52 & $0.19-1.45$ & & 0.64 & $0.29-1.41$ & \\
\hline \multicolumn{14}{|l|}{ Year of allo-HCT } \\
\hline 2004-2011 & 56 & Ref & & .71 & Ref & & .53 & Ref & & .41 & Ref & & .98 \\
\hline $2012-2017$ & 54 & 1.12 & $0.58-2.17$ & & 0.83 & $0.45-1.54$ & & 0.67 & $0.26-1.70$ & & 0.92 & $0.43-1.97$ & \\
\hline \multicolumn{14}{|c|}{ Female donor to male recipient } \\
\hline Yes & 18 & Ref & & .23 & Ref & & .53 & Ref & & .97 & Ref & & .47 \\
\hline No & 92 & 0.64 & $0.30-1.35$ & & 0.79 & $0.38-1.65$ & & 0.97 & $0.28-3.35$ & & 0.72 & $0.31-1.70$ & \\
\hline \multicolumn{14}{|l|}{ ABO compatibility } \\
\hline Compatible & 61 & Ref & & .61 & Ref & & .97 & Ref & & .50 & Ref & & .52 \\
\hline Minor & 18 & 0.85 & $0.32-2.26$ & & 1.08 & $0.47-2.52$ & & 2.02 & $0.68-5.94$ & & 0.44 & $0.10-1.88$ & \\
\hline Major & 20 & 1.51 & $0.69-3.28$ & & 1.17 & $0.55-2.51$ & & 1.01 & $0.28-3.68$ & & 1.22 & $0.48-3.08$ & \\
\hline Bidirectional & 11 & 1.44 & $0.54-3.85$ & & 1.17 & $0.45-3.06$ & & 0.57 & $0.08-4.29$ & & 1.56 & $0.53-4.59$ & \\
\hline \multicolumn{14}{|l|}{ Donor type } \\
\hline Related & 51 & Ref & & .001 & Ref & & .002 & Ref & & .12 & Ref & & $<.001$ \\
\hline MUD 10/10 & 44 & 1.46 & $0.72-2.95$ & & 2.35 & $1.21-4.55$ & & 2.33 & $0.87-6.21$ & & 1.77 & $0.76-4.14$ & \\
\hline$<10 / 10$ & 15 & 3.96 & $1.76-8.88$ & & 3.64 & $1.63-8.15$ & & 0.57 & $0.07-4.90$ & & 5.64 & $2.20-14.45$ & \\
\hline \multicolumn{14}{|c|}{ DIPSS classification at allo-HCT } \\
\hline Low-intermediate-1 & 26 & Ref & & .023 & Ref & & .035 & Ref & & .16 & Ref & & .22 \\
\hline Intermediate-2 to high & 83 & 2.60 & $1.09-6.18$ & & 2.18 & $1.01-4.70$ & & 2.69 & $0.62-11.66$ & & 1.65 & $0.70-3.91$ & \\
\hline \multicolumn{14}{|l|}{ Recipient CMV } \\
\hline Negative & 19 & Ref & & .46 & Ref & & .89 & Ref & & .98 & Ref & & .86 \\
\hline Positive & 91 & 1.39 & $0.58-3.30$ & & 1.06 & $0.49-2.26$ & & 1.02 & $0.29-3.55$ & & 1.07 & $0.41-2.83$ & \\
\hline \multicolumn{14}{|l|}{ Donor CMV } \\
\hline Negative & 49 & Ref & & .25 & Ref & & .18 & Ref & & .65 & Ref & & .28 \\
\hline Positive & 60 & 0.70 & $0.38-1.30$ & & 0.68 & $0.38-1.21$ & & 0.81 & $0.32-2.02$ & & 0.70 & $0.34-1.43$ & \\
\hline \multicolumn{14}{|l|}{ KPS } \\
\hline $90-100$ & 46 & Ref & & .36 & Ref & & .43 & Ref & & .057 & Ref & & .65 \\
\hline $70-80$ & 37 & 1.45 & $0.71-2.94$ & & 1.39 & $0.70-2.77$ & & 1.95 & $0.56-6.78$ & & 1.07 & $0.46-2.48$ & \\
\hline Unknown & 27 & 0.86 & $0.37-1.97$ & & 1.54 & $0.75-3.17$ & & 3.96 & $1.21-12.99$ & & 0.74 & $0.29-1.88$ & \\
\hline HCT-CI & & & & & & & & & & & & & \\
\hline 0 & 38 & Ref & & .59 & Ref & & .41 & Ref & & .085 & Ref & & .73 \\
\hline 1 & 26 & 1.48 & $0.65-3.37$ & & 1.47 & $0.65-3.33$ & & 1.97 & $0.45-8.60$ & & 1.20 & $0.46-3.13$ & \\
\hline
\end{tabular}

HCT-Cl, hematopoietic cell transplantation-specific comorbidity index; KPS, Karnofsky performance status; Ref, reference.

*Based on univariable analysis and log-rank test.

tBased on cumulative incidence accounting for competing risks and Gray test. 
Table 2. (continued)

\begin{tabular}{|c|c|c|c|c|c|c|c|c|c|c|c|c|c|}
\hline \multirow[b]{2}{*}{ Characteristic } & \multirow[b]{2}{*}{ No. } & \multicolumn{3}{|c|}{ os } & \multicolumn{3}{|c|}{ DFS } & \multicolumn{3}{|c|}{ Relapse } & \multicolumn{3}{|c|}{ NRM } \\
\hline & & $\mathbf{H R}^{*}$ & $95 \% \mathrm{Cl}$ & $\boldsymbol{P}^{*}$ & $\mathbf{H R}^{*}$ & $95 \% \mathrm{Cl}$ & $\boldsymbol{P}^{*}$ & HRt & $95 \% \mathrm{Cl}$ & $P+$ & HRt & $95 \% \mathrm{Cl}$ & $P+$ \\
\hline 2 & 21 & 1.51 & $0.61-3.73$ & & 1.68 & $0.71-3.98$ & & 1.89 & $0.38-9.33$ & & 1.43 & $0.51-4.04$ & \\
\hline Unknown & 25 & 0.96 & $0.40-2.28$ & & 1.85 & $0.85-4.02$ & & 4.70 & $1.25-17.60$ & & 0.88 & $0.33-2.38$ & \\
\hline \multicolumn{14}{|l|}{ MF } \\
\hline Primary & 58 & Ref & & .29 & Ref & & .13 & Ref & & .77 & Ref & & .19 \\
\hline Secondary & 52 & 0.72 & $0.39-1.34$ & & 0.64 & $0.36-1.15$ & & 0.87 & $0.35-2.19$ & & 0.61 & $0.29-1.27$ & \\
\hline \multicolumn{14}{|l|}{ Ruxolitinib use } \\
\hline No & 78 & Ref & & .33 & Ref & & .96 & Ref & & .26 & Ref & & .35 \\
\hline Yes & 32 & 1.39 & $0.68-2.86$ & & 0.98 & $0.49-1.96$ & & 0.49 & $0.14-1.73$ & & 1.40 & $0.61-3.22$ & \\
\hline \multicolumn{14}{|c|}{ Splenectomy before HCT } \\
\hline No & 94 & Ref & & .24 & Ref & & .82 & Ref & & .015 & Ref & & .062 \\
\hline Yes & 16 & 0.58 & $0.23-1.50$ & & 1.09 & $0.50-2.37$ & & 3.31 & $1.28-8.60$ & & 0.31 & $0.07-1.38$ & \\
\hline \multicolumn{14}{|l|}{ Cytogenetics } \\
\hline Favorable & 67 & Ref & & .006 & Ref & & .004 & Ref & & .035 & Ref & & .23 \\
\hline Unfavorable & 24 & 1.80 & $0.84-3.86$ & & 1.29 & $0.62-2.69$ & & 0.54 & $0.12-2.46$ & & 1.80 & $0.75-4.34$ & \\
\hline VHR & 15 & 3.11 & $1.48-6.53$ & & 3.00 & $1.49-6.04$ & & 2.95 & $1.11-7.82$ & & 1.99 & $0.81-4.90$ & \\
\hline
\end{tabular}

HCT-Cl, hematopoietic cell transplantation-specific comorbidity index; KPS, Karnofsky performance status; Ref, reference.

*Based on univariable analysis and log-rank test.

tBased on cumulative incidence accounting for competing risks and Gray test.

favorable long-term survival of 75\% in adverse-risk (MIPSS70+ v2.0 high and VHR risk) patients with MF who underwent allo-HCT.

Our study is the first and largest single-center experience using
Flu/Mel-based RIC for allo-HCT in MF patients, and it is among the first studies to characterize the underlying molecular and cytogenetic changes in patients undergoing allo-HCT.

Table 3. Multivariable analysis of associations of baseline characteristics and clinical outcomes

\begin{tabular}{|c|c|c|c|c|c|c|c|}
\hline \multirow[b]{2}{*}{ Characteristic } & \multirow[b]{2}{*}{ No. } & \multicolumn{3}{|c|}{ os } & \multicolumn{3}{|c|}{ DFS } \\
\hline & & Adjusted HR* & $95 \% \mathrm{Cl}$ & $\boldsymbol{P}^{*}$ & Adjusted $\mathbf{H R}^{\star}$ & $95 \% \mathrm{Cl}$ & $\boldsymbol{P}^{*}$ \\
\hline \multicolumn{8}{|l|}{ Sex } \\
\hline Male & 65 & Ref & & .005 & Ref & & .008 \\
\hline Female & 45 & 0.37 & $0.18-0.74$ & & 0.41 & $0.21-0.79$ & \\
\hline \multicolumn{8}{|l|}{ Donor type } \\
\hline MRD & 51 & Ref & & $<.001$ & Ref & & $<.001$ \\
\hline MUD 10/10 & 44 & 1.43 & $0.70-2.95$ & & 2.38 & $1.21-4.68$ & \\
\hline mMUD & 15 & 8.32 & $3.47-19.93$ & & 6.30 & $2.69-14.74$ & \\
\hline \multicolumn{8}{|l|}{ MF } \\
\hline Primary & 58 & Ref & & .094 & Ref & & .024 \\
\hline Secondary & 52 & 0.56 & $0.29-1.10$ & & 0.48 & $0.26-0.91$ & \\
\hline \multicolumn{8}{|l|}{ Cytogenetics } \\
\hline Favorable & 67 & Ref & & .001 & Ref & & .008 \\
\hline Unfavorable & 24 & 2.31 & $1.05-5.07$ & & 1.39 & $0.66-2.94$ & \\
\hline VHR & 15 & 4.19 & $1.93-9.13$ & & 3.14 & $1.53-6.47$ & \\
\hline \multicolumn{8}{|c|}{ MIPSS70+ v2.0 classification } \\
\hline Intermediate risk & 11 & 0.29 & $0.04-2.26$ & $<.001$ & 0.24 & $0.03-1.91$ & $<.001$ \\
\hline High risk & 47 & Ref & & & Ref & & \\
\hline VHR & 35 & 5.05 & $2.39-10.70$ & & 3.87 & $1.90-7.88$ & \\
\hline
\end{tabular}

*Based on the multivariable Cox regression model adjusting for sex, donor type, and cytogenetics.

tBased on the multivariable Cox regression model adjusting for sex and donor type, but not for cytogenetics. 


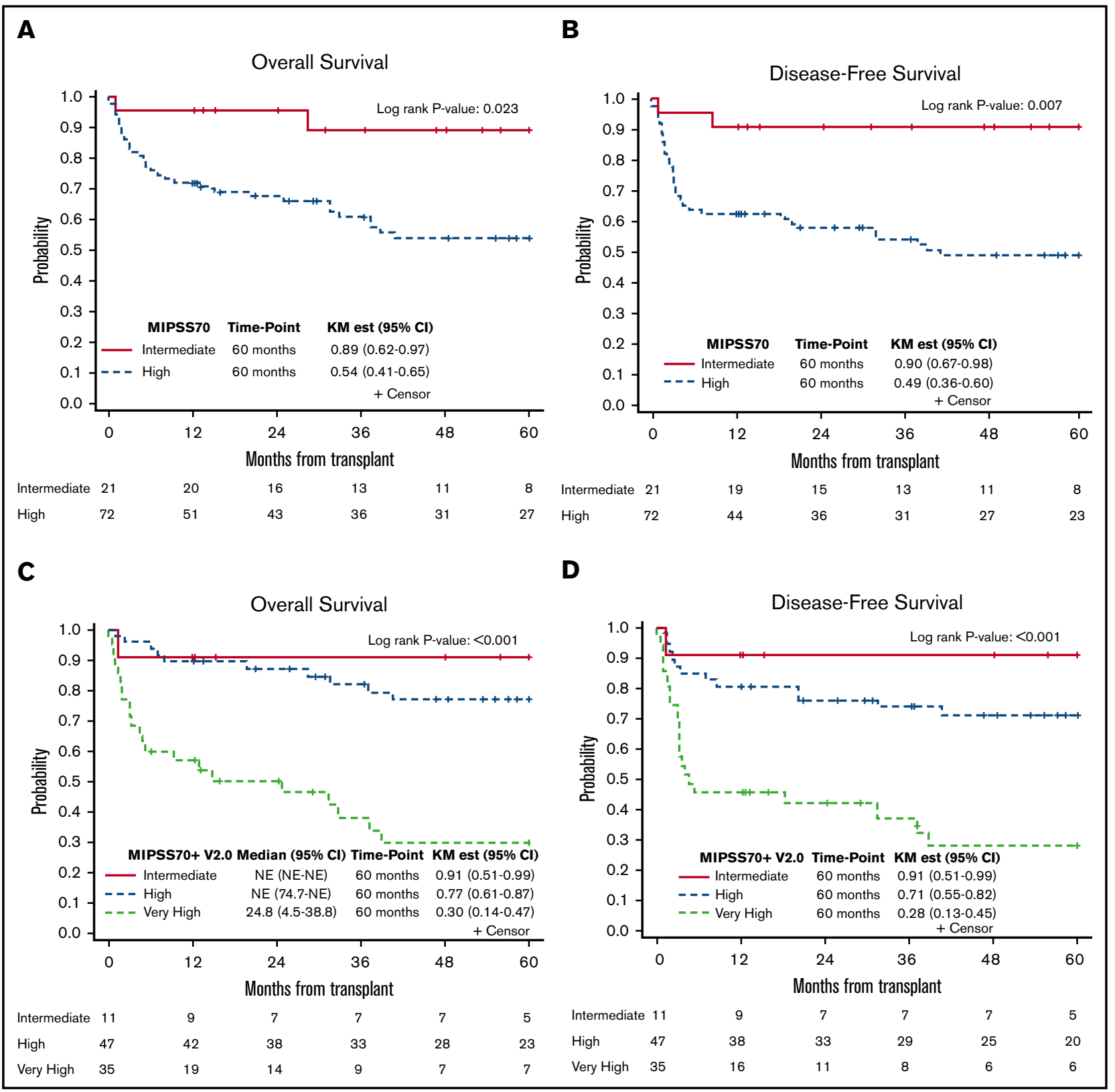

Figure 3. Kaplan-Meier curves demonstrating survival outcomes at 60 months after allo-HCT. OS (A) and DFS (B) in intermediate-risk and high-risk patients per MIPSS70 classification; OS (C) and DFS (D) in intermediate-risk, high-risk, and VHR patients per MIPSS70+ v2.0 classification. NE, not estimated.

Our OS data are similar to what has been reported previously by other investigators using RIC before allo-HCT. ${ }^{9,10,13}$ However, the relapse rate in our study was only $17 \%$ at 5 years (vs previously reported rates of $29 \%$ to $48 \%),{ }^{9,10}$ which might be attributable to the semiablative Flu/Mel conditioning. ${ }^{15,24}$ It is also possible that using sirolimus for GVHD prophylaxis had a positive impact on disease control because everolimus, a similar mTOR inhibitor, demonstrated a promising efficacy when tested as a single agent in a phase $1 / 2$ study of MF. ${ }^{25}$

The impact of somatic mutations has been extensively studied in nontransplantation settings. But no studies have evaluated the impact of these mutations on transplant outcomes, except for a recent report by Kroger et al. ${ }^{5}$ In contrast to the earlier study, which used a focused 16-gene panel, we used a more comprehensive NGS panel (developed at our center) that covered 72 genes, which allowed us to better characterize the mutation landscape of this disease.

Inconsistent with the earlier report by Kroger et al, ${ }^{5} C A L R, A S X L 1$, and $I D H$ mutations did not have an impact on transplantation outcomes in our study. These differences could be explained by the protective effect of the Flu/Mel regimen and/or Tac/Sir GVHD prophylaxis as discussed above, or by the lack of sufficient numbers of patients to reach statistical significance. Similarly, triple-negative, HMR, and $>1 \mathrm{HMR}$ status, which are reported to 
be associated with poor OS and leukemia-free survival in nontransplant patients, ${ }^{6,26}$ did not have an impact on survival or relapse in our cohort. Only $C B L$ mutations were associated with increased NRM in our cohort. Several studies of patients with MF have indicated an association between $C B L$ mutation and reduced leukemia-free survival and $O S$ outside the transplantation setting. ${ }^{27-30} C B L$ genes encode a negative regulator of JAK2 signaling, and gain-of-function mutations in $C B L$ lead to loss of its tumor suppressor function. Thus, it may play a role during leukemic transformation. ${ }^{30}$ However, the number of patients with $C B L$ mutations was small; therefore, a larger cohort needs to be evaluated to determine the definite impact of this mutation on transplant outcomes.

Recently Tefferi et $\mathrm{al}^{31}$ reported that allogeneic transplant could overcome the detrimental survival effect of unfavorable or VHR karyotype (per DIPSS) in 67 patients with MF, contrary to our findings, in which VHR was associated with higher risk of relapse. These differences could be explained by having a more homogeneous conditioning regimen and GVHD prophylactic regimen in our study compared with 4 different conditioning regimens, including myeloablative regimen in $18 \%$ of patients and 3 different GVHD prophylaxis regimens in Tefferi's study.

Detailed molecular characterizations and better understanding of cytogenetic risks has allowed further refinement of prognostic scoring systems originally derived from clinical factors (IPSS, Lille Scoring System, and DIPSS). ${ }^{32}$ Specifically, the MIPSS70 prognostic system was developed to predict outcomes of patients age 70 years or younger. ${ }^{1}$ That prognostic system was recently revised as MIPSS70+ v2.0, and it had refinements in degrees of anemia, cytogenetics, and HMR. ${ }^{7}$ Our study is the first to apply these newly developed molecularly integrated prognostic systems in HCT recipients. In our cohort, 77\% of evaluable patients were classified as high risk by MIPSS70, and $83 \%$ of patients were classified as high risk or VHR by MIPSS70+ v2.0. Although DIPSS classification was not predictive for transplant outcomes in our cohort, both MIPSS70 and MIPSS70+ v2.0 classifications predicted OS and DFS. Furthermore, MIPSS70+ v2.0 classification was associated with NRM, but MIPSS70 classification was not, supporting the idea that the high-risk patients by MIPSS70+ v2.0 require additional considerations regarding their candidacy for transplantation and novel approaches to reduce NRM.

Currently, allo-HCT is recommended for younger MF patients who have good performance status and organ functions, an available suitable donor, and a DIPSS risk profile of intermediate-2 or high. ${ }^{33}$ However, for individual patients, discussions and decision-making processes are complex and need to be individualized. In fact, the Centers for Medicare \& Medicaid Services released a decision memo in 2016 allowing "coverage with evidence development" allo-HCT in their beneficiaries for MF, recognizing that there is insufficient evidence about the qualitative and quantitative benefits of allo-HCT. Our cohort included 25 patients $(22 \%)$ who were age 65 years or older; about $50 \%$ of patients were age 60 years or older. Our data, which demonstrate a favorable long-term survival after allo-HCT and predictive capability of MIPSS70 + v2.0, will contribute to discussions and decisionmaking processes for allo-HCT in MF patients.

Our data on survival after allo-HCT in high-risk and VHR groups by MIPSS70 + 2.0 are clearly better than the data for patients who did not receive a transplant reported previously by Tefferi et al, ${ }^{34}$ which showed a 5 -year OS of $30 \%$ in VHR patients. Even though there is an inherent and strong selection bias, our data support the notion that allo-HCT should be considered and offered for these high-risk MF patients. It is important to note that in our cohort, a significant number of patients who were categorized as having low or intermediate risk by DIPSS were reassigned to higher risk by MIPSS70 and higher risk or VHR by MIPSS70 + v2.0, showing that the DIPSS might not be sufficient for stratifying these patients. Thus, to obtain better outcomes for patients with MF, results of our study suggest stratification based on MIPSS70+ v2.0 and allogeneic transplantation for higher-risk patients.

In summary, despite the inherent limitations resulting from the retrospective nature of our analysis, our results indicate that alloHCT with the Flu/Mel regimen and Tac/Sir GVHD prophylaxis is associated with favorable long-term OS, DFS, low relapse rate, and acceptable NRM in patients with primary or secondary MF. This is the first study to demonstrate that MIPSS70 can predict outcome after allo-HCT in this population and thus would better inform physicians and patients for discussing and decision making about alloHCT. Our data also suggest that novel approaches to reduce complications and the risk of relapse are needed for MIPSS70 high-risk populations. Such novel approaches may include the peri-HCT use of ruxolitinib (NCT02917096), which is being explored in a study currently underway at our institution.

\section{Acknowledgments}

The authors thank Ketevan Gendzekhadze from City of Hope HLA laboratory for assistance with sample collection, Barbara Choi from City of Hope Pathology for technical assistance, and City of Hope staff, nurses, and the patients and their families, without whom this work would not be possible.

Research reported in this publication included work performed in the Bioinformatics and Pathology Cores supported by the National Cancer Institute of the National Institutes of Health under award number P30CA033572.

The content is solely the responsibility of the authors and does not necessarily represent the official views of the National Institutes of Health.

\section{Authorship}

Contribution: H.A., I.A., R.N., R.P., and D.S. designed and performed research, analyzed the data, and wrote the manuscript with input and supervision from S.K., A.S.S., G.M., and S.J.F.; D.Y. analyzed data; S.M. helped write and edit the manuscript; and A.A., M.A., M.M.A.M., T.C., M.M., M.O., A.S., V.P., and L.Y. discussed the results and contributed to the final version of the manuscript.

Conflict-of-interest disclosure: The authors declare no competing financial interests.

ORCID profiles: H.A., 0000-0002-9728-7292; M.M., 00000002-1109-6955.

Correspondence: Haris Ali, Department of Hematology and Hematopoietic Cell Transplantation, City of Hope National Medical Center, 1500 E Duarte Rd, Duarte, CA 91010; e-mail: harisali@coh. org. 


\section{References}

1. Arber DA, Orazi A, Hasserjian R, et al. The 2016 revision to the World Health Organization classification of myeloid neoplasms and acute leukemia. Blood. 2016;127(20):2391-2405.

2. Cervantes F, Dupriez B, Pereira A, et al. New prognostic scoring system for primary myelofibrosis based on a study of the International Working Group for Myelofibrosis Research and Treatment. Blood. 2009;113(13):2895-2901.

3. Dupriez B, Morel P, Demory JL, et al. Prognostic factors in agnogenic myeloid metaplasia: a report on 195 cases with a new scoring system. Blood. 1996; 88(3):1013-1018.

4. Vainchenker W, Kralovics R. Genetic basis and molecular pathophysiology of classical myeloproliferative neoplasms. Blood. 2017;129(6):667-679.

5. Kröger N, Panagiota V, Badbaran A, et al. Impact of molecular genetics on outcome in myelofibrosis patients after allogeneic stem cell transplantation. Biol Blood Marrow Transplant. 2017;23(7):1095-1101.

6. Tefferi A, Lasho TL, Finke CM, et al. CALR vs JAK2 vs MPL-mutated or triple-negative myelofibrosis: clinical, cytogenetic and molecular comparisons. Leukemia. 2014;28(7):1472-1477.

7. Guglielmelli P, Lasho TL, Rotunno G, et al. MIPSS70: Mutation-Enhanced International Prognostic Score System for transplantation-age patients with primary myelofibrosis. J Clin Oncol. 2018;36(4):310-318.

8. Tefferi A, Nicolosi M, Mudireddy M, et al. Revised cytogenetic risk stratification in primary myelofibrosis: analysis based on 1002 informative patients. Leukemia. 2018;32(5):1189-1199.

9. Kröger N, Holler E, Kobbe G, et al. Allogeneic stem cell transplantation after reduced-intensity conditioning in patients with myelofibrosis: a prospective, multicenter study of the Chronic Leukemia Working Party of the European Group for Blood and Marrow Transplantation. Blood. 2009;114(26): 5264-5270.

10. Gupta V, Malone AK, Hari PN, et al. Reduced-intensity hematopoietic cell transplantation for patients with primary myelofibrosis: a cohort analysis from the center for international blood and marrow transplant research. Biol Blood Marrow Transplant. 2014;20(1):89-97.

11. Ballen KK, Shrestha S, Sobocinski KA, et al. Outcome of transplantation for myelofibrosis. Biol Blood Marrow Transplant. 2010;16(3):358-367.

12. Lussana F, Rambaldi A, Finazzi MC, et al. Allogeneic hematopoietic stem cell transplantation in patients with polycythemia vera or essential thrombocythemia transformed to myelofibrosis or acute myeloid leukemia: a report from the MPN Subcommittee of the Chronic Malignancies Working Party of the European Group for Blood and Marrow Transplantation. Haematologica. 2014;99(5):916-921.

13. Rondelli D, Goldberg JD, Isola L, et al. MPD-RC 101 prospective study of reduced-intensity allogeneic hematopoietic stem cell transplantation in patients with myelofibrosis. Blood. 2014;124(7):1183-1191.

14. Gupta V, Hari P, Hoffman R. Allogeneic hematopoietic cell transplantation for myelofibrosis in the era of JAK inhibitors. Blood. 2012;120(7):1367-1379.

15. Aldoss I, Pham A, Li SM, et al. Favorable impact of allogeneic stem cell transplantation in patients with therapy-related myelodysplasia regardless of TP53 mutational status. Haematologica. 2017;102(12):2030-2038.

16. Li MM, Datto M, Duncavage EJ, et al. Standards and Guidelines for the Interpretation and Reporting of Sequence Variants in Cancer: A Joint Consensus Recommendation of the Association for Molecular Pathology, American Society of Clinical Oncology, and College of American Pathologists. $J$ Mol Diagn. 2017;19(1):4-23.

17. Richards S, Aziz N, Bale S, et al; ACMG Laboratory Quality Assurance Committee. Standards and guidelines for the interpretation of sequence variants: a joint consensus recommendation of the American College of Medical Genetics and Genomics and the Association for Molecular Pathology. Genet Med. 2015;17(5):405-424.

18. Przepiorka D, Weisdorf D, Martin P, et al. 1994 consensus conference on acute GVHD grading. Bone Marrow Transplant. 1995;15(6):825-828.

19. Jagasia MH, Greinix HT, Arora M, et al. National Institutes of Health Consensus Development Project on Criteria for Clinical Trials in Chronic Graft-versusHost Disease: I. The 2014 Diagnosis and Staging Working Group report. Biol Blood Marrow Transplant. 2015;21 (3):389-401.e1.

20. Holtan SG, DeFor TE, Lazaryan A, et al. Composite end point of graft-versus-host disease-free, relapse-free survival after allogeneic hematopoietic cell transplantation. Blood. 2015;125(8):1333-1338.

21. Tefferi A, Lasho TL, Jimma T, et al. One thousand patients with primary myelofibrosis: the mayo clinic experience. Mayo Clin Proc. 2012;87(1):25-33.

22. Snyder DS, Palmer J, Gaal K, et al. Improved outcomes using tacrolimus/sirolimus for graft-versus-host disease prophylaxis with a reduced-intensity conditioning regimen for allogeneic hematopoietic cell transplant as treatment of myelofibrosis. Biol Blood Marrow Transplant. 2010;16(2):281-286.

23. Snyder DS, Palmer J, Stein AS, et al. Allogeneic hematopoietic cell transplantation following reduced intensity conditioning for treatment of myelofibrosis. Biol Blood Marrow Transplant. 2006;12(11):1161-1168.

24. Al Malki MM, Nathwani N, Yang D, et al. Melphalan-based reduced-intensity conditioning is associated with favorable disease control and acceptable toxicities in patients older than 70 with hematologic malignancies undergoing allogeneic hematopoietic stem cell transplantation. Biol Blood Marrow Transplant. 2018;24(9):1828-1835.

25. Guglielmelli P, Barosi G, Rambaldi A, et al; AIRC-Gruppo Italiano Malattie Mieloproliferative (AGIMM) investigators. Safety and efficacy of everolimus, a mTOR inhibitor, as single agent in a phase 1/2 study in patients with myelofibrosis. Blood. 2011;118(8):2069-2076.

26. Guglielmelli P, Lasho TL, Rotunno G, et al. The number of prognostically detrimental mutations and prognosis in primary myelofibrosis: an international study of 797 patients. Leukemia. 2014;28(9):1804-1810.

27. Tefferi A, Lasho TL, Finke CM, et al. Targeted deep sequencing in primary myelofibrosis. Blood Adv. 2016;1(2):105-111. 
28. Bose P, Verstovsek S. Prognosis of primary myelofibrosis in the genomic era. Clin Lymphoma Myeloma Leuk. 2016;16(Suppl):S105-S113.

29. Beer PA, Delhommeau F, LeCouédic JP, et al. Two routes to leukemic transformation after a JAK2 mutation-positive myeloproliferative neoplasm. Blood. 2010;115(14):2891-2900.

30. Sanada M, Suzuki T, Shih LY, et al. Gain-of-function of mutated C-CBL tumour suppressor in myeloid neoplasms. Nature. 2009;460(7257):904-908.

31. Tefferi A, Partain DK, Palmer JM, et al. Allogeneic hematopoietic stem cell transplant overcomes the adverse survival effect of very high risk and unfavorable karyotype in myelofibrosis. Am J Hematol. 2018;93(5):649-654.

32. Gangat N, Caramazza D, Vaidya R, et al. DIPSS plus: a refined Dynamic International Prognostic Scoring System for primary myelofibrosis that incorporates prognostic information from karyotype, platelet count, and transfusion status. J Clin Oncol. 2011;29(4):392-397.

33. Tefferi A. Primary myelofibrosis: 2017 update on diagnosis, risk-stratification, and management. Am J Hematol. 2016;91(12):1262-1271.

34. Tefferi A, Guglielmelli P, Lasho TL, et al. MIPSS70+ Version 2.0: Mutation and Karyotype-Enhanced International Prognostic Scoring System for Primary Myelofibrosis. J Clin Oncol. 2018;36(17):1769-1770. 